\title{
On the oxygen abundance in our Galaxy
}

\author{
L. S. Pilyugin ${ }^{1}$, F. Ferrini ${ }^{2}$, and R. V. Shkvarun ${ }^{1}$ \\ 1 Main Astronomical Observatory of National Academy of Sciences of Ukraine, 27 Zabolotnogo str., 03680 Kiev, Ukraine \\ e-mail: shkvarun@mao.kiev.ua \\ 2 Department of Physics, Section of Astronomy, University of Pisa, piazza Torricelli 2, 56100 Pisa, Italy \\ e-mail: federico.ferrini@df.unipi.it
}

Received 12 November 2002 / Accepted 9 January 2003

\begin{abstract}
The compilation of published spectra of Galactic $\mathrm{H}$ II regions with available diagnostic [OIII] $\lambda 4363$ line information has been carried out. Our list contains 71 individual measurements of $13 \mathrm{H}$ II regions in the range of galactocentric distances from 6.6 to $14.8 \mathrm{kpc}$. The oxygen abundances in all the $\mathrm{H}_{\text {II }}$ regions were recomputed in the same way, using the classic $T_{\mathrm{e}}$-method. The oxygen abundance at the solar galactocentric distance traced by those $\mathrm{H}_{\text {II }}$ regions is in agreement with the oxygen abundance in the interstellar medium in the solar vicinity derived with high precision from the interstellar absorption lines towards stars. The derived radial oxygen abundance distribution was compared with that for H II regions from the Shaver et al. (1983) sample which is the basis of many models for the chemical evolution of our Galaxy. It was found that the original Shaver et al.'s oxygen abundances are overestimated by $0.2-0.3$ dex. Oxygen abundances in H II regions from the Shaver et al. sample have been redetermined with the recently suggested $P$-method. The radial distribution of oxygen abundances from the Shaver et al. sample redetermined with the $P$-method is in agreement with our radial distribution of $(\mathrm{O} / \mathrm{H})_{T_{\mathrm{e}}}$ abundances.
\end{abstract}

Key words. galaxies: abundances - ISM: abundances - ISM: general - galaxies: individual: Milky Way Galaxy

\section{Introduction}

By now, spectra have been obtained for hundreds of $\mathrm{H}$ II regions in spiral galaxies. Accurate oxygen abundances can be derived from measurement of temperature-sensitive line ratios, such as [OIII]4959,5007/[OIII]4363. This method will be referred to as the $T_{\mathrm{e}}$-method. Unfortunately, in oxygen-rich $\mathrm{H}_{\text {II }}$ regions the temperature-sensitive lines such as [OIII]4363 are too weak to be detected. For such $\mathrm{H}$ II regions, empirical abundance indicators based on more readily observable lines were suggested (Pagel et al. 1979; Alloin et al. 1979). The empirical oxygen abundance indicator $R_{23}=([\mathrm{OII}] 3727,3729+$ [OIII $] 4959,5007) / \mathrm{H}_{\beta}$, suggested by Pagel et al. (1979), has found widespread acceptance and use for the oxygen abundance determination in $\mathrm{H}$ II regions where the temperaturesensitive lines are undetectable. This method will be referred to as the $R_{23}$-method. Using the $R_{23}$-method, the characteristic oxygen abundances (the oxygen abundance at a predetermined galactocentric distance) and radial oxygen abundance gradients were obtained for a large sample of spiral galaxies (Vila-Costas \& Edmunds 1992; Zaritsky et al. 1994; van Zee et al. 1998, among others).

It has been found (Pilyugin 2001b) that the $(\mathrm{O} / \mathrm{H})_{T_{\mathrm{e}}}$ data is sufficient in quantity and quality for an accurate determination of the value of the oxygen abundance gradient within the

Send offprint requests to: L. S. Pilyugin, e-mail: pilyugin@mao.kiev.ua disk of the spiral galaxy M 101. It has been found that the parameters of the $(\mathrm{O} / \mathrm{H})_{R_{23}}$ abundance distribution in the disk of M 101 differ significantly from those of the $(\mathrm{O} / \mathrm{H})_{T_{\mathrm{e}}}$ abundance distribution. The rather low value of the central oxygen abundance $12+\log (\mathrm{O} / \mathrm{H})_{T_{\mathrm{e}}}(R=0)=8.81$ in the disk of $\mathrm{M} 101$ was found. This is in agreement with the result of Kinkel \& Rosa (1994). They carried out special spectral observastions of one single high-metallicity $\mathrm{H}_{\text {II }}$ region in the disk of the spiral galaxy M 101 (NGC 5457) and were able to derive the electron temperature, allowing the determination of the oxygen abundance with the classic $T_{\mathrm{e}}$-method. Kinkel \& Rosa (1994) found that the oxygen abundance derived with the $T_{\mathrm{e}}$-method is lower by around 0.2 dex with respect to the oxygen abundance obtained on the basis of the $R_{23}$ calibration after Edmunds \& Pagel (1984). Castellanos et al. (2002) have also found that the $R_{23}$-method results in the overestimated oxygen abundances in the case of low-excitation $\mathrm{H}_{\text {II }}$ regions.

Pilyugin et al. (2002) have re-determined the oxygen abundances in $\mathrm{H}_{\mathrm{II}}$ regions in a number of field and Virgo spiral galaxies with the $P$-method. It has been found for the considered sample of galaxies that the central values $12+$ $\log (\mathrm{O} / \mathrm{H})_{P}(R=0)$ lie in the range $8.71 \div 8.99$ while the central values $12+\log (\mathrm{O} / \mathrm{H})_{R_{23}}(R=0)$ lie in the range $9.24 \div 9.53$. Thus, for all the galaxies, in which the oxygen abundances in H II regions were derived with the $T_{\mathrm{e}}$ - or with the $P$-method, the value of the central oxygen abundance does not exceed $12+\log (\mathrm{O} / \mathrm{H})(R=0) \sim 9.0$. 
The radial distribution of oxygen and other element abundances in our Galaxy derived by Shaver et al. (1983) from consideration of $\mathrm{H}$ II regions has found wide acceptance. The Shaver et al.'s data are the basis of the models for the chemical evolution of the Milky Way Galaxy constructed by different investigators (Tosi 1988; Giovagnoli \& Tosi 1995; Thon \& Meusinger 1998, among others). Shaver et al. (1983) have found the value of the central oxygen abundance in our Galaxy as large as $12+\log (\mathrm{O} / \mathrm{H})(R=0)=9.38 \pm 0.04$. Radio recombination lines have been used by Shaver et al. to determine electron temperatures in $\mathrm{H}$ II regions and these temperatures have been applied to optical spectra of the same $\mathrm{H}_{\text {II }}$ regions to determine the abundances of oxygen and other elements. The Galactic $\mathrm{H}$ II regions with measured temperature-sensitive line ratios will be considered in the present study, and, as consequence, the radial distribution of the $(\mathrm{O} / \mathrm{H})_{T_{\mathrm{e}}}$ abundances within the disk of the Milky Way Galaxy will be established. The comparison of the obtained radial distribution of the $(\mathrm{O} / \mathrm{H})_{T_{\mathrm{e}}}$ abundances with that from Shaver et al. (1983) allows us to verify the credibility of the oxygen abundances derived by Shaver et al. (1983).

\section{2. $\mathrm{H}$ II regions with available [OIII] $\lambda 4363$ line intensity}

Spectroscopic observations of Galactic $\mathrm{H}$ II regions have been carried out by many investigators (Baldwin et al. 1991, 2000; Caplan et al. 2000; Peimbert et al. 1969, 1977, 1978, 1992, 1993; Esteban 1999; Esteban et al. 1998, 1999; Osterbrock et al. 1992; Shaver et al. 1983; Vílchez \& Esteban 1996, among others). The Galactic H II regions with measured temperaturesensitive line ratios are listed in Table 1.

Spectroscopic data with detections of diagnostic emission lines in the $\mathrm{H}_{\text {II }}$ region makes it possible to determine an accurate oxygen abundance $(\mathrm{O} / \mathrm{H})_{T_{\mathrm{e}}}$. However, the oxygen abundances in the same $\mathrm{H}_{\mathrm{II}}$ region with measured line ratios [OIII] $\lambda \lambda 4959,5007 / \lambda 4363$ derived in different works can differ due to different atomic data adopted and different interpretation of the temperature structure (e.g. single characteristic $T_{\mathrm{e}}$, two-zone model for $T_{\mathrm{e}}$, model with smallscale temperature fluctuations). Therefore the compilation of $\mathrm{H}$ II regions with original oxygen abundance determinations through the $T_{\mathrm{e}}$-method from different works carried out over more than thirty years is not a set of homogeneous determinations. Accordingly, the available published spectra of $\mathrm{H}$ II regions with measured line ratios [OIII] $\lambda \lambda 4959,5007 / \lambda 4363$ have been reanalysed to produce a homogeneous set. Two-zone models of $\mathrm{H}$ II regions with the algorithm for oxygen abundance determination from Pagel et al. (1992) and the $T_{\mathrm{e}}([\mathrm{OII}])-$ $T_{\mathrm{e}}([\mathrm{OIII}])$ relation from Garnett (1992) were adopted here,

$$
\begin{aligned}
\frac{\mathrm{O}}{\mathrm{H}}=\frac{\mathrm{O}^{+}}{\mathrm{H}^{+}}+\frac{\mathrm{O}^{++}}{\mathrm{H}^{+}}, & \\
12+\log \left(\mathrm{O}^{++} / \mathrm{H}^{+}\right)= & \log \frac{I_{[\mathrm{OIII}] \lambda 4959+\lambda 5007}}{I_{\mathrm{H}_{\beta}}}+6.174 \\
& +\frac{1.251}{t_{3}}-0.55 \log t_{3},
\end{aligned}
$$

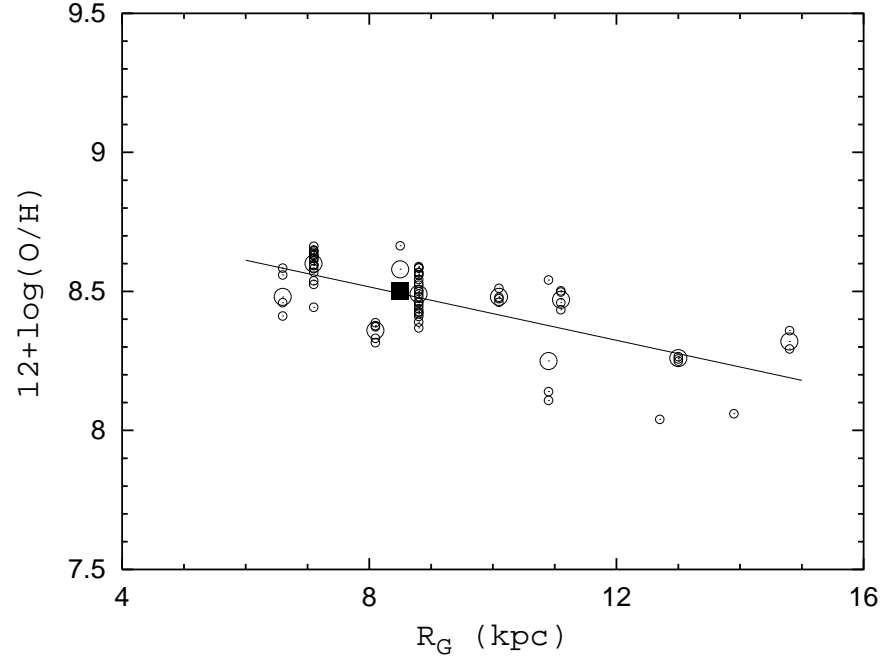

Fig. 1. Oxygen abundance in Galactic $\mathrm{H}_{\text {II }}$ regions with an available [OIII] $\lambda 4363$ line intensity as a function of galactocentric distance. Small open circles are oxygen abundances $(\mathrm{O} / \mathrm{H})_{T_{\mathrm{e}}}$ for individual measurements, the solid line is the best fit to those data. Large open circles are mean values of oxygen abundances $\left\langle(\mathrm{O} / \mathrm{H})_{T_{\mathrm{e}}}\right\rangle$ in $\mathrm{H}_{\text {II }}$ regions. The large filled square is the oxygen abundance in the interstellar gas in the solar vicinity derived from the high-resolution observations of the weak interstellar OI $\lambda 1356$ absorption lines towards stars.

$$
\begin{aligned}
12+\log \left(\mathrm{O}^{+} / \mathrm{H}^{+}\right)= & \log \frac{I_{[\mathrm{OII}] \lambda 3726+\lambda 3729}}{I_{\mathrm{H}_{\beta}}}+5.890 \\
& +\frac{1.676}{t_{2}}-0.40 \log t_{2}+\log (1+1.35 x), \\
t_{3}=t([\mathrm{OIII}])= & 1.432\left[\log \frac{I_{[\mathrm{OIII}] \lambda 4959+\lambda 5007}}{I_{[\mathrm{OIII}] \lambda 4363}}-0.85\right. \\
& \left.+0.03 \log t_{3}+\log \left(1+0.0433 x t_{3}^{0.06}\right)\right]^{-1}
\end{aligned}
$$

$x=10^{-4} n_{\mathrm{e}} t_{2}^{-1 / 2}$,

where $n_{\mathrm{e}}$ is the electron density in $\mathrm{cm}^{-3}$ (the value of $n_{\mathrm{e}}$ was adopted to be equal to $100 \mathrm{~cm}^{-3}$ for every $\mathrm{H}_{\text {II }}$ region), $t_{3}=t_{[\mathrm{OIII}]}$ is the electron temperature within the [OIII] zone in units of $10^{4} \mathrm{~K}, t_{2}=t_{[\mathrm{OII}]}$ is the electron temperature within the [OII] zone in units of $10^{4} \mathrm{~K}$. The $t_{2}$ value is determined from the equation (Garnett 1992)

$t_{2}=0.7 t_{3}+0.3$.

The electron temperatures $T_{\mathrm{e}}([\mathrm{OIII}])$ in $\mathrm{H}$ II regions (with two exceptions, $\mathrm{H}$ II regions $\mathrm{S} 127$ and $\mathrm{S} 128$ ) have been determined from the measurements of [OIII] $\lambda \lambda 4959,5007 / \lambda 4363$ line ratios, and the electron temperatures $T_{\mathrm{e}}([\mathrm{OII}])$ in $\mathrm{H}_{\mathrm{II}}$ regions have been derived from the $T_{\mathrm{e}}([\mathrm{OII}])-T_{\mathrm{e}}([\mathrm{OIII}])$ relation of Garnett (1992). In the case of $\mathrm{H}_{\text {II }}$ regions $\mathrm{S} 127$ and S 128 the electron temperatures $T_{\mathrm{e}}([\mathrm{OIII}])$ cannot be directly determined from observational data since the measurement of the [OIII] $\lambda \lambda 4959,5007 / \lambda 4363$ line ratio is not available. Instead, the temperature-sensitive lines [OII] $\lambda \lambda 7320,7330$ were detected in spectrophotometry of $\mathrm{H}$ II regions $\mathrm{S} 127$ and S 128 (Vílchez \& Esteban 1996). The original oxygen abundances in these $\mathrm{H}$ II regions are given in Table 1. 
Table 1. Oxygen abundance (mean value $\left\langle(\mathrm{O} / \mathrm{H})_{T_{\mathrm{e}}}\right\rangle$ ) in Galactic $\mathrm{H}_{\text {II }}$ regions with available [OIII] $\lambda 4363$ line intensity. List of references: 1 - Esteban et al. (1999); 2 - Peimbert \& Costero (1969); 3 - Peimbert et al. (1993); 4 - Caplan et al. (2000); 5 - Deharveng et al. (2000); 6 - Esteban (1999); 7 - Peimbert et al. (1992); 8 - Peimbert et al. (1978); 9 - Baldwin et al. (1991); 10 - Baldwin et al. (2000); 11 - Esteban et al. (1998); 12 - Osterbrock et al. (1992); 13 - Peimbert et al. (1977); 14 - Shaver et al. (1983); 15 - Vílchez \& Esteban (1996).

\begin{tabular}{lccll}
\hline \hline H II region & $\begin{array}{c}\text { galacto- } \\
\text { centric } \\
\text { distance } \\
\text { kpc }\end{array}$ & $\begin{array}{c}\text { number } \\
\text { of } \\
\text { measu- } \\
\text { rements }\end{array}$ & ref. & $(\mathrm{O} / \mathrm{H})_{T_{\mathrm{e}}}^{*}$ \\
\hline M 8 & 6.6 & 3 & $1,2,3$ & \\
M 16 & 6.6 & 1 & 4,5 & $8.48 \pm 0.06$ \\
M 17 & 7.1 & 18 & $2,6,7$ & $8.60 \pm 0.05$ \\
Carina & 8.1 & 5 & 8 & $8.36 \pm 0.03$ \\
Sh 117 & 8.5 & 2 & 4,5 & $8.58 \pm 0.08$ \\
Orion & 8.8 & 24 & $2,4,5,9-13$ & $8.49 \pm 0.06$ \\
Sh 184 & 10.1 & 4 & 4,5 & $8.48 \pm 0.02$ \\
Sh 311 & 10.9 & 3 & 8,14 & $8.26 \pm 0.20$ \\
Sh 206 & 11.1 & 4 & 4,5 & $8.47 \pm 0.03$ \\
Sh 128 & 12.7 & 1 & 15 & $8.04 \pm 0.20^{1}$ \\
Sh 298 & 13.0 & 3 & 8,14 & $8.26 \pm 0.01$ \\
Sh 127 & 13.9 & 1 & 15 & $8.06 \pm 0.20^{1}$ \\
Sh 212 & 14.8 & 2 & 4,5 & $8.32 \pm 0.04$ \\
\hline
\end{tabular}

* In units of $12+\log (\mathrm{O} / \mathrm{H})$.

${ }^{1}$ Original data.

In Fig. 1 we show oxygen abundances $(\mathrm{O} / \mathrm{H})_{T_{\mathrm{e}}}$ for the individual measurements as a function of galactocentric distance with small open circles. The galactocentric distances are from Deharveng et al. (2000). The best fit to the individual $(\mathrm{O} / \mathrm{H})_{T_{\mathrm{e}}}$ data is

$$
12+\log (\mathrm{O} / \mathrm{H})_{T_{\mathrm{e}}}=8.90-0.048 R_{\mathrm{G}}
$$

The scatter is equal to 0.10 dex. Equation (7) results in the oxygen abundance at a solar galactocentric distance as large as $12+\log (\mathrm{O} / \mathrm{H}) \simeq 8.50$. The $(\mathrm{O} / \mathrm{H})_{T_{\mathrm{e}}}-R_{\mathrm{G}}$ relationship is shown in Fig. 1 by a solid line. The large open circles in Fig. 1 are average oxygen abundances $(\mathrm{O} / \mathrm{H})_{T_{\mathrm{e}}}$ for $\mathrm{H}$ II regions (the data from Table 1).

Thus, the obtained value of the central oxygen abundance in the disk of the Milky Way Galaxy $12+\log (\mathrm{O} / \mathrm{H})_{T_{\mathrm{e}}}(R=0)=8.90$ lies in the same range as the values of the central oxygen abundance in the disks of other spiral galaxies, in which the oxygen abundances in $\mathrm{H}_{\text {II }}$ regions were derived with the $T_{\mathrm{e}}$ - or with the $P$-method.

\section{The oxygen abundance in the solar vicinity}

The above obtained radial distributions of $(\mathrm{O} / \mathrm{H})_{T_{\mathrm{e}}}($ Eq. (7)) results in the oxygen abundance at a solar galactocentric distance as large as $12+\log (\mathrm{O} / \mathrm{H}) \simeq 8.50$. Let us compare this value with recent determinations by other investigators.
Caplan et al. (2000) and Deharveng et al. (2000) have analysed Galactic $\mathrm{H}_{\text {II }}$ regions and have obtained the slope $-0.0395 \mathrm{dex} / \mathrm{kpc}$ with a central oxygen abundance $12+$ $\log (\mathrm{O} / \mathrm{H})=8.82$ and $12+\log (\mathrm{O} / \mathrm{H})=8.48$ at the solar galactocentric distance. Rodríguez (1999) considered seven bright Galactic $\mathrm{H}_{\text {II }}$ regions with galactocentric distances in the range 6-10 kpc and has found that all the $\mathrm{H}$ in regions studied are characterized by similar abundances, $12+\log (\mathrm{O} / \mathrm{H}) \sim 8.45 \pm 0.1$. Thus, our value of the oxygen abundance at the solar galactocentric distance is in agreement with that derived recently in other studies.

High-resolution observations of the weak interstellar OI $\lambda 1356$ absorption towards stars allow one to determine interstellar gas-phase oxygen abundance in the solar vicinity with very high precesion. These observations yield a mean interstellar gas-phase oxygen abundance of $319 \mathrm{O}$ atoms per $10^{6} \mathrm{H}$ atoms (or $12+\log (\mathrm{O} / \mathrm{H})=8.50$ ) (Meyer et al. 1998; Sofia \& Meyer 2001). The interstellar gas-phase oxygen abundance in the solar vicinity is shown in Fig. 1 by the large filled square. There are no statistically significant variations in the measured oxygen abundances from line of sight to line of sight; the rms scatter value for these oxygen abundances is low, \pm 0.05 dex. Out to $1.5 \mathrm{kpc}$, the gas-phase oxygen abundances are stable in diffuse clouds with different physical conditions as measured by the fraction of $\mathrm{H}$ in the form of $\mathrm{H}_{2}$. Thus, our value of the oxygen abundance at the solar galactocentric distance derived from consideration of the $\mathrm{H}_{\text {II }}$ regions is in agreement with that derived with high precision from the interstellar absorption towards the stars, Fig. 1.

The oxygen abundances in $\mathrm{H}$ II regions were determined within the framework of two-zone model for $T_{\mathrm{e}}$ within the H II region. The agreement between the value of the oxygen abundance at the solar galactocentric distance derived from consideration of the $\mathrm{H}$ II regions and that derived from the interstellar absorption towards stars can be considered as strong evidence in favor that the two-zone model for $T_{\mathrm{e}}$ is a realistic interpretation of the temperature structure within H II regions. It should be noted however that this argument is not indisputable due to the dust-phase oxygen abundance. Indeed, one can expect that total (gas- plus dust-phase) oxygen abundances in the $\mathrm{H}$ II regions are equal to the total oxygen abundance in the interstellar medium. The gas-phase oxygen abundances in the $\mathrm{H}_{\text {II }}$ regions are expected to be equal to the gas-phase oxygen abundance in the interstellar medium if only the same fraction of oxygen is incorporated into dust grains both in $\mathrm{H}_{\text {II }}$ regions and in the interstellar medium. Meyer et al. (1998) have obtained the limit to the dust-phase oxygen abundance in the interstellar medium in the vicinity of the Sun. Assuming various mixtures of oxygen-bearing grain compounds, they found that it is difficult to increase the oxygen dust fraction beyond $120 \mathrm{O}$ atoms per $10^{6} \mathrm{H}$ atmos, simply because the requisite metals are far less abundant than oxygen (if $\mathrm{Si} / \mathrm{O}, \mathrm{Mg} / \mathrm{O}$ and $\mathrm{Fe} / \mathrm{O}$ abundance ratios in the present-day interstellar medium are the same as they are in the Sun). Then the upper value of total (gas- plus dust-phase) oxygen abundance in the interstellar medium in the vicinity of the Sun is of $439 \mathrm{O}$ atoms per million $\mathrm{H}$ atoms (or $12+\log (\mathrm{O} / \mathrm{H})=8.64$ ). Then, in the case of the dust-free $\mathrm{H}_{\text {II }}$ regions the gas-phase 
oxygen abundances in the $\mathrm{H}$ II regions in the vicinity of the Sun would be equal to the total oxygen abundance and would be as large as $12+\log (\mathrm{O} / \mathrm{H})=8.64$. In this case the gas-phase oxygen abundances in $\mathrm{H}$ II regions, determined within the framework of two-zone model for $T_{\mathrm{e}}$, would be underestimated by 0.14 dex. Taking into account that $\mathrm{H}$ II regions are not dust-free (according to Esteban et al. 1998, the fraction of the dust-phase oxygen abundance in the Orion nebula is about $0.1 \mathrm{dex}$ ) one can expect that the gas-phase oxygen abundances in the $\mathrm{H}$ in regions are close (or equal) to the gas-phase oxygen abundance in the interstellar medium, and, consequently, one can conclude that two-zone model for $T_{\mathrm{e}}$ distribution within the $\mathrm{H}$ II region provides a realistic gas-phase oxygen abundance in $\mathrm{H}$ II regions.

It has been known for a long time that permitted lines in $\mathrm{H}$ II regions indicate higher oxygen abundances than forbidden lines. Esteban et al. (1998) found oxygen abundances for two positions in the Orion nebula from permitted and forbidden lines. The oxygen abundances derived from forbidden lines are coincident for both positions in the Orion nebula $(12+\log (\mathrm{O} / \mathrm{H})=8.47)$ and agree well with the interstellar oxygen abundance in the solar vicinity derived from interstellar absorption lines towards the stars $(12+\log (\mathrm{O} / \mathrm{H})=$ 8.50). They found from permitted lines the oxygen abundance $12+\log (\mathrm{O} / \mathrm{H})=8.61$ for position 1 and $12+\log (\mathrm{O} / \mathrm{H})=8.68$ for position 2 , although the abundances obtained from the different multiplets observed show significant dispersion. If permitted lines indicate the true oxygen abundance in the Orion nebula, then the uncertainty around $0.1 \mathrm{dex}$ in the oxygen abundances derived from forbidden lines cannot be excluded. However, the origin of the discrepancy between abundances derived from permitted and forbidden lines is not indisputable (see discussion in Stasinska 2002).

Hence the value of total (gas- plus dust-phase) oxygen abundance in the interstellar medium in the vicinity of the Sun is $12+\log (\mathrm{O} / \mathrm{H})=8.6 \pm 0.1$. Young stars provide an excellent method for probing the metallicity and recent evolutionary history of the Milky Way Galaxy. Early B-type dwarfs have evolutionary ages typically less than $30 \mathrm{Myr}$ and possess photospheres that are not normally contaminated by core-processed material. Hence, these stars should provide information on the present-day chemical composition of the interstellar medium within the Milky Way Galaxy. There have been several attempts to measure the oxygen abundance using young, B-type stars as tracers of metallicity in the Galactic disk (Fitzsimmons et al. 1992; Kaufer et al. 1994; Smartt \& Rolleston 1977; Gummersbach et al. 1998; Daflon et al. 1999; Rolleston et al. 2000; Daflon et al. 2001, among others). Unfortunately, results from stars are controversial. Recently, Rolleston et al. (2000) and Daflon et al. (2001) have derived oxygen abundances in early type stars using both LTE and non-LTE calculations (see the discussion of earlier results in Rolleston et al. 2000). Rolleston et al. (2000) have found from early type stars a $12+\log (\mathrm{O} / \mathrm{H})$ value of around 8.80 for the solar vicinity, while Daflon et al. (2001) have found from a non-LTE analysis a $12+\log (\mathrm{O} / \mathrm{H})$ value around 8.50 for the solar vicinity region. It should be taken into account however that Rolleston et al. (2000) have noted that their data should be reliable for estimating the magnitude of the metal abundance gradients in the present-day Galactic disk; although, the absolute abundance values at any particular galactocentric distance may be in error.

Andrievsky et al. (2002) have derived the radial distribution of the oxygen (and other chemical elements) abundance within the Galactic disk based on the spectroscopic analysis of a sample of classic Cepheids. They have found from Cepheids an oxygen abundance gradient in the Galactic disk that results in a $[\mathrm{O} / \mathrm{H}]$ value of around -0.07 dex for the solar vicinity region. Andrievsky et al. (2002) have derived a differential abundances in the sence that the oscillator strengths used in their study were obtained through an inverted solar analysis (with adopted solar abundances). As consequence, their absolute oxygen abundance values depend on the adopted solar oxygen abundance. The recent determinations of the solar oxygen abundance result in the value of $12+\log (\mathrm{O} / \mathrm{H})_{\odot} \sim 8.70$ (Holweger 2001; Prieto et al. 2001), i.e. the present-day "recommended" solar abundance is less (by more than $0.2 \mathrm{dex}$ ) than the value recommended several years ago (Anders \& Grevesse 1989; Grevesse et al. 1996; Grevesse \& Sauval 1998). With this recent solar oxygen abundance, the data of Andrievsky et al. (2002) result in a $12+\log (\mathrm{O} / \mathrm{H})$ value of around 8.60 for the solar vicinity.

Thus, the value of the oxygen abundance at the solar galactocentric distance $(12+\log (\mathrm{O} / \mathrm{H}) \sim 8.6)$ derived by Andrievsky et al. (2002) from the study of Cepheids and the value of the oxygen abundance $(12+\log (\mathrm{O} / \mathrm{H}) \sim 8.5)$ derived by Daflon et al. $(1999,2001)$ from the study of OB stars are in agreement with the oxygen abundance in the solar vicinity region derived from the interstellar absorption towards stars and from the study of $\mathrm{H}$ II regions. At the same time, there is an appreciable disagreement between values of the radial oxygen abundance gradient derived from the study of Cepheids $(-0.022 \mathrm{dex} / \mathrm{kpc}$, Andrievsky et al. 2002), from the study of early B-type mainsequence stars $(-0.067 \mathrm{dex} / \mathrm{kpc}$, Rolleston et al. 2000), and from the study of $\mathrm{H}$ II regions $(-0.048 \mathrm{dex} / \mathrm{kpc}$, this study). This disagreement can be explained by two reasons. First, there is a relatively large scatter of oxygen abundance values at any particular galactocentric distance for objects of any kind (Cepheids, OB stars, and $\mathrm{H}_{\mathrm{II}}$ regions). Second. A majority of data-points, used in the determination of the value of the radial oxygen abundance gradient, are distributed over a restricted $\left(6<R_{\mathrm{G}}<13 \mathrm{kpc}\right)$ range of galactocentric distances: all the Cepheids from the sample of Andrievsky et al. have galactocentric distances in the range 6-11 kpc, only three objects from the sample of Rolleston et al. exist for galactocentric distances greater than $R_{\mathrm{G}} \sim 13 \mathrm{kpc}$, only three individual measurements of two $\mathrm{H}_{\text {II }}$ regions with galactocentric distances greater than $R_{\mathrm{G}} \sim 13 \mathrm{kpc}$ were used in this study. This disagreement prevents us from reaching a definitive conclusion about the value of the radial oxygen abundance gradient within the Galactic disk; though we stress that more and better data are needed to derive the exact value of gradient.

It should be noted that the recent low value of the solar oxygen abundance still exceeds slightly the present-day total oxygen abundance in the interstellar medium at the solar galactocentric distance (see also Daflon et al. 2001). If it is the case, this is evidence that the variation of oxygen abundance at the solar galactocentric distance with time is not smooth (for example, due to an irregular rate of unenriched gas infall onto 


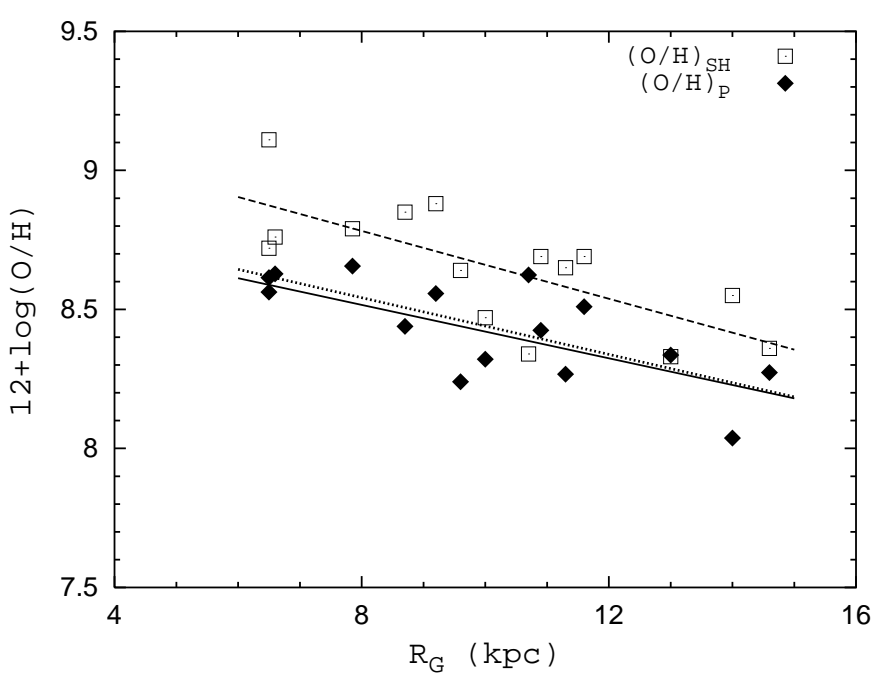

Fig. 2. Oxygen abundance in Galactic H II regions from sample of Shaver et al. (1983) as a function of galactocentric distance. The galactocentric distances are from Deharveng et al. (2000). The open squares are original oxygen abundances $(\mathrm{O} / \mathrm{H})_{\mathrm{SH}}$ from Shaver et al. (1983), the dashed line is the best fit to those data. The filled rhombuses are oxygen abundances $(\mathrm{O} / \mathrm{H})_{P}$ derived here with the $P$-method using the same measurements of the line intensities, the dotted line is the best fit to those data. The solis line is the $(\mathrm{O} / \mathrm{H})_{T_{\mathrm{e}}}-R_{\mathrm{G}}$ relationship (see Fig. 1).

the disk, see Pilyugin \& Edmunds 1996). The discussion of the model for the chemical evolution of our Galaxy is far beyond the goal of the present study, we only would like to note that the recent low solar oxygen abundance results in better agreement with the present-day total oxygen abundance in the interstellar medium (and in young stars) at the solar galactocentric distance than generally accepted earlier solar values.

\section{Shaver et al.'s set of $\mathrm{H}$ II regions}

Combining radio and optical spectroscopy Shaver et al. (1983) have found chemical abundances in a large sample of Galactic $\mathrm{H}_{\text {II }}$ regions covering a wide range in galactocentric radius. Radio recombination lines have been used to determine electron temperatures in $\mathrm{H}_{\text {II }}$ regions and these temperatures have been applied to optical spectra of the same $\mathrm{H}_{\text {II }}$ regions to determine the abundances of oxygen and other elements. Shaver et al. (1983) have found that their oxygen abundances are in agreement with the empirical $R_{23}$-calibration. At the same time it has been found (Pilyugin 2000, 2001a,b, 2003) that the oxygen abundances derived with the $R_{23}$-calibration after Edmunds $\&$ Pagel (1984) involve a systematic error depending on the excitation parameter: the $R_{23}$-method provides more or less realistic oxygen abundances in high-excitation $\mathrm{H}_{\text {II }}$ regions and yields an overestimated oxygen abundances in low-excitation $\mathrm{H}$ II regions. Taking into account that many $\mathrm{H}$ II regions in the sample of Shaver et al. (1983) are low-excitation objects, the agreement of Shaver et al's oxygen abundances with the empirical $R_{23}$-calibration hints that their oxygen abundances are overestimated.

Recently a new way of oxygen abundance determination in $\mathrm{H}_{\text {II }}$ regions, in which the physical conditions in $\mathrm{H}_{\text {II }}$ region are estimated via the excitation parameter $P$ ( $P$-method), was
Table 2. Oxygen abundances in set of $\mathrm{H}_{\text {II }}$ regions from Shaver et al. (1983). The name of $\mathrm{H}_{\text {II }}$ region is given in Col. 1. The galactocentric distance taken from Deharveng et al. (2000) is reported in Col. 2. The original oxygen abundance $(\mathrm{O} / \mathrm{H})_{\mathrm{SH}}$ from Shaver et al. (1983) is listed in Col. 3. The oxygen abundance $(\mathrm{O} / \mathrm{H})_{P}$ determined here with the $P$-method is reported in Col. 4.

\begin{tabular}{lccc}
\hline \hline H II region & $\begin{array}{c}\text { galactocentric } \\
\text { distance } \\
(\mathrm{kpc})\end{array}$ & $\begin{array}{c}(\mathrm{O} / \mathrm{H})_{\mathrm{SH}}^{*} \\
\text { Shaver et al. } \\
(1983)\end{array}$ & $\begin{array}{c}(\mathrm{O} / \mathrm{H})_{P}^{*} \\
\text { this work }\end{array}$ \\
\hline NGC 6604-1 & 6.5 & 9.11 & 8.61 \\
NGC 6604-2 & 6.5 & 8.72 & 8.56 \\
M 16 & 6.6 & 8.76 & 8.63 \\
1 Cen-2 & 7.8 & 8.79 & 8.66 \\
RCW 40 & 8.7 & 8.85 & 8.44 \\
RCW 34 & 9.2 & 8.88 & 8.56 \\
RCW 19 & 9.6 & 8.64 & 8.24 \\
Rosette-1 & 10.0 & 8.47 & 8.32 \\
S 252-1 & 10.7 & 8.34 & 8.62 \\
RCW 16-1 & 10.9 & 8.69 & 8.42 \\
RCW 8 & 11.3 & 8.65 & 8.27 \\
RCW 6 & 11.6 & 8.69 & 8.51 \\
RCW 5-1 & 13.0 & 8.33 & 8.34 \\
G 201.6+1.6 & 14.0 & 8.55 & 8.04 \\
S 284 & 14.6 & 8.36 & 8.27 \\
\hline
\end{tabular}

${ }^{*}$ In units of $12+\log (\mathrm{O} / \mathrm{H})$.

suggested (Pilyugin 2001a). By comparing the oxygen abundances in high-metallicity $\mathrm{H}_{\mathrm{II}}$ regions derived with the $T_{\mathrm{e}^{-}}$ method and those derived with the $P$-method, it was found that the precision of oxygen abundance determination with the $P$-method is comparable to that of the $T_{\mathrm{e}}$-method (Pilyugin $2001 \mathrm{a}, \mathrm{b})$. Then the comparison of oxygen abundances obtained by Shaver et al. (1983) and oxygen abundances determined with the $P$-method using the same line intensities can give some idea of the accuracy of Shaver et al's oxygen abundances. The oxygen abundances obtained by Shaver et al. (1983) will be referred to as $(\mathrm{O} / \mathrm{H})_{\mathrm{SH}}$ abundances, the oxygen abundances derived with the $P$-method will be referred to as $(\mathrm{O} / \mathrm{H})_{P}$ abundances. Only $\mathrm{H}_{\mathrm{II}}$ regions with reliable measured ratios $[\mathrm{OII}]_{3727,3729} / \mathrm{H}_{\beta}$ and $[\mathrm{OIII}]_{4959,5007} / \mathrm{H}_{\beta}$ from the sample of Shaver et al. (1983) will be considered. These $\mathrm{H}$ II regions are listed in Table 2. $(\mathrm{O} / \mathrm{H})_{\mathrm{SH}}$ abundances as a function of galactocentric distance are shown in Fig. 2 by the open squares. The linear best fit to those data is presented by the dashed line in Fig. 2. The galactocentric distances are from Deharveng et al. (2000). The best fit to $(\mathrm{O} / \mathrm{H})_{\mathrm{SH}}$ abundances is given by the following equation

$12+\log (\mathrm{O} / \mathrm{H})_{\mathrm{SH}}=9.27-0.061 R_{\mathrm{G}}$

The scatter is equal to 0.15 dex. Equation (8) results in the oxygen abundance at the solar galactocentric distance as large as $12+\log (\mathrm{O} / \mathrm{H})=8.75$. 
The $(\mathrm{O} / \mathrm{H})_{P}$ oxygen abundances in $\mathrm{H}_{\text {II }}$ regions are determined with the expression suggested in (Pilyugin 2001a)

$12+\log (\mathrm{O} / \mathrm{H})_{P}=\frac{R_{23}+54.2+59.45 P+7.31 P^{2}}{6.07+6.71 P+0.37 P^{2}+0.243 R_{23}}$,

where $R_{23}=R_{2}+R_{3}, R_{2}=I_{[\mathrm{OII}] \lambda 3727+\lambda 3729} / I_{\mathrm{H} \beta}, R_{3}=$ $I_{[\mathrm{OIII}] \lambda 4959+\lambda 5007} / I_{* \mathrm{H} \beta}$, and $P=R_{3} / R_{23}$. The $(\mathrm{O} / \mathrm{H})_{P}$ abundances in $\mathrm{H}$ II regions from the sample of Shaver et al. (1983) are listed in Col. 4 in Table 2. $(\mathrm{O} / \mathrm{H})_{P}$ abundances as a function of galactocentric distance are shown in Fig. 2 by the filled rhombuses. The linear best fit to those data is presented by the dotted line in Fig. 2. The best fit to $(\mathrm{O} / \mathrm{H})_{P}$ abundances is given by the following equation

$$
12+\log (\mathrm{O} / \mathrm{H})_{P}=8.95-0.051 R_{\mathrm{G}}
$$

The scatter is equal to 0.12 dex. Equation (10) results in the oxygen abundance at the solar galactocentric distance as large as $12+\log (\mathrm{O} / \mathrm{H})=8.52$.

Examination of Fig. 2 (see also the data from Table 2) shows that there is a systematic difference between $(\mathrm{O} / \mathrm{H})_{\mathrm{SH}}$ and $(\mathrm{O} / \mathrm{H})_{P}$ abundances, $(\mathrm{O} / \mathrm{H})_{\mathrm{SH}}$ abundances are higher on average by $0.2-0.3$ dex than $(\mathrm{O} / \mathrm{H})_{P}$ abundances. Taking into account that the precision of oxygen abundance determination with the $P$-method is comparable to that of the $T_{\mathrm{e}}$-method (Pilyugin 2001a,b) we can conclude that the Shaver et al.'s oxygen abundances are overestimated by $0.2-0.3$ dex.

Our conclusion can be verified by comparison of the radial $(\mathrm{O} / \mathrm{H})_{\mathrm{SH}}$ abundances distribution with the radial $(\mathrm{O} / \mathrm{H})_{T_{\mathrm{e}}}$ abundance distribution across the disk of the Milky Way Galaxy. (It should be stressed that the reliability of $(\mathrm{O} / \mathrm{H})_{T_{\mathrm{e}}}$ abundances in $\mathrm{H}$ II regions is confirmed by the agreement between the value of the oxygen abundance at the solar galactocentric distance traced by $(\mathrm{O} / \mathrm{H})_{T_{\mathrm{e}}}$ abundances in the $\mathrm{H}_{\mathrm{II}}$ regions and that derived from the interstellar absorption towards stars, a modelindependent method.) The $(\mathrm{O} / \mathrm{H})_{T_{\mathrm{e}}}-R_{\mathrm{G}}$ relationship obtained above is shown in Fig. 2 by a solid line. Inspection of Fig. 2 shows that the $(\mathrm{O} / \mathrm{H})_{\mathrm{SH}}$ abundances lie above (in average by $0.2-0.3 \mathrm{dex})$ the $(\mathrm{O} / \mathrm{H})_{T_{\mathrm{e}}}-R_{\mathrm{G}}$ relationship. This confirms our conclusion that the Shaver et al.'s oxygen abundances are overestimated by a factor of about 2 .

\section{Conclusions}

The compilation of spectra of Galactic $\mathrm{H}_{\text {II }}$ regions with available diagnostic [OIII] $\lambda 4363$ line was carried out. Our list contains 71 individual measurements of $13 \mathrm{H}$ II regions in the range of galactocentric distances from 6.6 to $14.8 \mathrm{kpc}$. The oxygen abundances in all the $\mathrm{H}_{\mathrm{II}}$ regions were recomputed in the same way, using the classic $T_{\mathrm{e}}$-method.

The oxygen abundance at the solar galactocentric distance traced by $\mathrm{H}$ II regions is in agreement with the oxygen abundance in the interstellar medium in the solar vicinity derived with high precision from the interstellar absorption lines towards stars.

The obtained value of the central oxygen abundance in the disk of the Milky Way Galaxy $12+\log (\mathrm{O} / \mathrm{H})_{T_{\mathrm{e}}}(R=0)=8.90$ lies in the same range as the values of the central oxygen abundance in the disks of other spiral galaxies, in which the oxygen abundances in $\mathrm{H}_{\text {II }}$ regions were derived with the $T_{\mathrm{e}}-$ or with the $P$-method.

The derived radial oxygen abundance distribution was compared with that for H II regions from the Shaver et al. (1983) sample which is the basis of many models for the chemical evolution of our Galaxy. It was found that the original Shaver et al.'s oxygen abundances are overestimated by $0.2-0.3$ dex. Oxygen abundances in $\mathrm{H}_{\text {II }}$ regions from the Shaver et al. sample have been redetermined with the recently suggested $P$-method. The radial distribution of oxygen abundances from the Shaver et al. sample redetermined with the $P$-method is in agreement with the radial distribution of $\mathrm{O} / \mathrm{H}_{T_{\mathrm{e}}}$ abundances obtained here.

Acknowledgements. We thank the anonymous referee for helpful comments. This study was partly supported by the Joint Research Project between Eastern Europe and Switzerland (SCOPE) No. 7UKPJ62178 (L.S.P.), the NATO grant PST.CLG.976036 (L.S.P. and F.F.), the Italian national grant delivered by the MURST (L.S.P.).

\section{References}

Alloin, D., Collin-Souffrin, S., Joly, M., \& Vigroux, L. 1979, A\&A, 78, 200

Anders, E., \& Grevesse, N. 1989, Geochim. Cosmochim. Acta, 53, 197

Andrievsky, S. M., Kovtyukh, V. V., Luck, R. E., et al. 2002, A\&A, 381,32

Baldwin, J. A., Ferland, G. J., Martin, P. G., et al. 1991, ApJ, 374, 580

Baldwin, J. A., Verner, E. M., Verner, D. A., et al. 2000, ApJS, 129, 229

Caplan, J., Deharveng, L., Peña, M., Costero, R., \& Blondel, C. 2000, MNRAS, 311, 328

Castellanos, M., Diáz, A. I., \& Terlevich, E. 2002, MNRAS, 329, 315

Daflon, S., Cunha, K., \& Becker, S. 1999, ApJ, 522, 950

Daflon, S., Cunha, K., Butler, K., \& Smith, V. V. 2001, ApJ, 563, 325

Deharveng, L., Peña, M., Caplan, J., \& Costero, R. 2000, MNRAS, 311,329

Edmunds, M. G., \& Pagel, B. E. J. 1984, MNRAS, 211, 507

Esteban, C. 1999, Rev. Mex. Astron. Astrofis., 35, 65

Esteban, C., Peimbert, M., Torres-Peimbert, S., \& Escalante, V. 1998, MNRAS, 295, 401

Esteban, C., Peimbert, M., Torres-Peimbert, S., García-Rojas, J., \& Rodríguez, M. 1999, ApJS, 120, 113

Fitzsimmons, A., Brown, P. J. F., \& Rolleston, W. R. J. 1992, MNRAS, 259,489

Garnett, D. R. 1992, AJ, 103, 1330

Giovagnoli, A., \& Tosi, M. 1995, MNRAS, 273, 499

Grevesse, N., Noels, A., \& Sauval, A. J. 1996, ASP Conf. Ser., 99, 117

Grevesse, N., \& Sauval, A. J. 1998, in Solar composition and its evolution - from core to corona, ed. C. Fröhlich, M. C. E. Huber, S. K. Solanki, \& R. vonSteiger (Netherlands: Kluwer Academic Publishers), Space Sci. Ser. ISSI, 5, 161

Gummersbach, C. A., Kaufer, A., Schäfer, D. R., Szeifert, T., \& Wolf, B. $1988, A \& A, 338,881$

Holweger, H. 2001, in Solar and Galactic Composition, ed. R. F. Wimmer - Schweingruber, American Institute of Physics Conf. Proc., 598, 23

Kaufer, A., Szeifert, T., Krenzin, R., Baschek, B., \& Wolf, B. 1994, A\&A, 289, 740

Kinkel, U., \& Rosa, M. R. 1994, A\&A, 282, L37

Meyer, D. M., Jura, M., \& Cardelli, J. A. 1998, ApJ, 493, 222 
Osterbrock, D. E., Tran, H. D., \& Veileux, S. 1992, ApJ, 389, 305

Pagel, B. E. J., Edmunds, M. G., Blackwell, D. E., Chun, M. S., \& Smith, G. 1979, MNRAS, 189, 95

Pagel, B. E. J., Simonson, E. A., Terlevich, R. J., \& Edmunds, M. G. 1992, MNRAS, 255, 325

Peimbert, M., \& Costero, R. 1969, Boletin de los observatorios de Tonanzintla y Tacubaya, 31, No. 5, 3

Peimbert, M., \& Torres-Peimbert, S. 1977, MNRAS, 179, 217

Peimbert, M., Torres-Peimbert, S., \& Rayo, J. F. 1978, ApJ, 220, 516

Peimbert, M., Torres-Peimbert, S., \& Ruiz, M. T. 1992, Rev. Mex. Astron. Astrofis., 24, 155

Peimbert, M., Torres-Peimbert, S., \& Dufour, R. J. 1993, ApJ, 418, 760

Pilyugin, L. S. 2000, A\&A, 358, 72

Pilyugin, L. S. 2001a, A\&A, 369, 594

Pilyugin, L. S. 2001b, A\&A, 373, 56

Pilyugin, L. S. 2003, A\&A, 399, 1003
Pilyugin, L. S., \& Edmunds, M. G. 1996, A\&A, 313, 792

Pilyugin, L. S., Mollá, M., Ferrini, F., \& Vílchez, J. M. 2002, A\&A, 383, 14

Prieto, C. A., Lambert, D. L., \& Asplund, M. 2001, ApJ, 556, L63

Rodríguez, M. 1999, A\&A, 351, 1075

Shaver, P. A., McGee, R. X., Newton, L. M., Danks, A. C., \& Pottasch, S. R. 1983, MNRAS, 204, 53

Smartt, S. J., \& Rolleston, W. R. J. 1997, ApJ, 481, L47

Sofia, U. J., \& Meyer, D. M. 2001, ApJ, 554, L221

Stasinska, G. 2002 [astro-ph/0207500]

Thon, R., \& Meusinger, H. 1998, A\&A, 338, 413

Tosi, M. 1988, A\&A, 197, 47

van Zee, L., Salzer, J. J., Haynes, M. P., O’Donoghue, A. A., \& Balonek, T. J. 1998, AJ, 116, 2805

Vila-Costas, M. B., \& Edmunds, M. G. 1992, MNRAS, 259, 121

Vílchez, J. M., \& Esteban, C. 1996, MNRAS, 280, 720

Zaritsky, D., Kennicutt, R. C., Jr., \& Huchra, J. P. 1994, ApJ, 420, 87 